Check for updates

Cite this: RSC Adv., 2019, 9, 21996

Received 27th May 2019

Accepted 11th July 2019

DOI: 10.1039/c9ra04003j

rsc.li/rsc-advances

\section{Efficient removal of crystal violet dye using EDTA/ graphene oxide functionalized corncob: a novel low cost adsorbent $\uparrow$}

\author{
Huan Wang, (D)* Xin Lai, Wei Zhao, Youning Chen, Xiaoling Yang, Xiaohua Meng \\ and Yuhong Li
}

In this study, EDTA functionalized corncob (EDTA-corncob) and EDTA/graphene oxide functionalized corncob (EDTA-GO/corncob) were prepared using disodium ethylenediamine tetraacetic acid and the graphene oxide immersion method. EDTA-corncob and EDTA-GO/corncob were characterized by SEM and FTIR spectroscopy. On this basis, the adsorption properties of EDTA-corncob and EDTA-GO/ corncob were studied with crystal violet as the adsorbate. The optimum adsorption conditions were determined by the effect of samples on the adsorption properties of crystal violet at different times, temperatures and $\mathrm{pH}$, and the reusability of the samples was studied. The results showed that adsorption capacity of crystal violet on EDTA-GO/corncob was higher compared with natural corncob and EDTAcorncob. The most suitable $\mathrm{pH}$ value of the solution is about 6.0, the adsorption equilibrium time is 200 min. EDTA-GO/corncob can be reused eight times. This study indicated that EDTA-GO/corncob is a reusable adsorbent for rapid, low-cost, and efficient removal of dye from waste water.

\section{Introduce}

Water pollution is one of the major environmental problems nowadays. ${ }^{1}$ Dyes are one of the important and basic pollutants in water pollution. With the accelerated process of industrialization development, more and more wastewater containing dyes is discharged from various human activities and causes serious threats to the environment. ${ }^{2-4}$

A wide range of physicochemical techniques, such as electrocoagulation, photocatalytic process, flocculation, ozonation, adsorption, and membrane filtration, have been employed to dispose of the wastewater. ${ }^{5-10}$ Among these various purification treatments, liquid-phase adsorption is believed to be the most effective way to remove organic dyes from wastewater. A variety of materials, which include chitosan, ${ }^{11}$ leaves,${ }^{12}$ crab shell, ${ }^{13}$ waste coal gangue,${ }^{14}$ peach gum polysaccharide,${ }^{15}$ ganoderma lucidum, ${ }^{16}$ clays, ${ }^{17}$ and Cucumis sativus peel, ${ }^{18}$ were prepared or used as adsorbents for removal of dyes from water.

Biomass waste, such as corncob, has great potential to form inexpensive and environmental friendly adsorbents due to the large quantities produced, chemical and mechanical stability, high surface area and structural properties. But generally the adsorption capacity of natural corncob is not ideal. Therefore, many

College of Chemistry and Chemical Engineering, Xianyang Normal University, Xianyang 712000,China. E-mail: 237463169@qq.com

$\dagger$ Electronic supplementary information (ESI) available: Fig. S1 The pseudo first-order kinetics model. Fig. S2 The pseudo second-order kinetics model. See DOI: $10.1039 / \mathrm{c} 9 \mathrm{ra} 04003 \mathrm{j}$ modification methods have been invented to increase the capacity of corncob based adsorbents. ${ }^{19,20} \mathrm{Ma}$ et al. reported that corncob was converted into a novel magnetic adsorbent (MCA) for removal of anionic and cationic dyes. ${ }^{19}$ Mesoporous activated carbon that was prepared from corncob was used as adsorbent for removal of ammonium from groundwater. ${ }^{20}$ Hyperbranched polyamide modified corncob was synthesized and used as adsorbent for $\mathrm{Cr}(\mathrm{vI})$ with maximum adsorption capacity that could reach up to $131.6 \mathrm{mg} \mathrm{g}^{-1}$. $^{21}$ EDTA is a kind of complexing agent, EDTAmodified may produce adsorbents with strong metal-complexing property, thus, EDTA-modified materials have been widely used for heavy metal and dyes removal, such as bamboo activated carbon, ${ }^{22} \beta$-cyclodextrin/chitosan, ${ }^{23}$ electrospun polyacrylonitrile nanofibers. ${ }^{24}$ Since the graphene was first reported in 2004, it has been widely investigated due to its excellent electrical conductivity, thermal stability, mechanical strength and adsorption capacity. ${ }^{25,26}$

In this study, in addition to introducing EDTA onto corncob for enhancing adsorption capacity, graphene oxide would be also added. EDTA functionalized corncob (EDTA-corncob) and EDTA/graphene oxide functionalized corncob (EDTA-GO/ corncob) were prepared successfully used to remove various dyes from water. Methylene blue, crystal violet, acridine orange, methyl red, acid chrome blue K, rhodamine B and orange IV, as typical anionic and cationic dye, were selected as the pollutants. At meantime, to fully understand the adsorption behavior of EDTA-corncob and EDTA-GO/corncob, studies were carried out under various parameters such as adsorbent dosage, $\mathrm{pH}$, contact time. Adsorption kinetics of EDTA-corncob and EDTA$\mathrm{GO} /$ corncob were investigated by pseudo first order model 
and pseudo second order model, respectively. Furthermore, the equilibrium data of EDTA-corncob and EDTA-GO/corncob were analyzed by Langmuir, Freundlich and Sips model, respectively. The reusability on adsorption capacity of EDTA-corncob and EDTA-GO/corncob were also tested. Our results demonstrate that EDTA-GO/corncob is a cheap and efficient adsorbent for removal of dye in aqueous media.

\section{Experimental section}

\subsection{Materials}

Flake graphite (99.95\%) was supplied by Qingdao Chenyang Graphite Co., Ltd. (Qingdao, China). Corncob was purchased from Deep Processing of Huifeng Straw Agricultural Products (Lianyungang, China). Ethylenediamine tetraacetic acid, were purchased from the National Medicine Group Chemical Reagent Co., Ltd. (Shanghai, China). Acridine orange, methyl red, crystal violet, rhodamine $\mathrm{B}$, orange IV, acidic chrome blue $\mathrm{K}$ and methylene blue were purchased from the Aladdin Chemical Reagent Co., Ltd. (Shanghai, China), and the other reagents were of analytical grade.

GO was prepared from the flake graphite powder using a modified Hummers-Offeman method. ${ }^{27}$

\subsection{Preparation of adsorbent}

2.2.1 Pretreatment of corncob. The corncobs were washed with tap water and distilled water carefully to remove surface impurities and dried at $55{ }^{\circ} \mathrm{C}$. Then the dried corncobs were crushed by a grinder to obtain corncob powder for storage.

$10.0 \mathrm{~g}$ of corncob powder was immersed in $20 \%$ isopropanol solution, stirred for 24 hours, filtered, washed with $20 \%$ isopropanol and distilled water to be colorless, dried for 24 hours at $55{ }^{\circ} \mathrm{C}$, then added $0.1 \mathrm{~mol} \mathrm{~L}{ }^{-1}$ of sodium hydroxide solution to the sample, stirred for 1 hour at room temperature, filtered, then added distilled water to stir for 45 minutes, washed repeatedly to $\mathrm{pH} 7.0$, and filtered. The obtained products were dried at $55{ }^{\circ} \mathrm{C}$ for storage.

2.2.2 Preparation of EDTA functionalized corncob/ graphene oxide. The pretreated corncob powder was soaked in $2.5 \%$ EDTA solution for 24 hours, filtered and washed with distilled water, and dried at $55{ }^{\circ} \mathrm{C}$. The obtained products were EDTA-corncob.

$0.10 \mathrm{~g}$ of GO was dispersed in $2.5 \%$ EDTA solution under ultrasound. Then the pretreated corncob powder was soaked in $2.5 \%$ EDTA/GO solution. After 24 hours, it was filtered and washed with distilled water. The EDTA-GO/corncob sample was dried at $55{ }^{\circ} \mathrm{C}$ and stored for reserve.

\subsection{Characterization of materials}

Fourier infrared spectrometry (FT-IR) of GO, corncob, EDTAcorncob and EDTA-GO/corncob were obtained using a Nicolet iS10 FT-IR spectrophotometer (Thermo Fisher Scientific, USA). Scanning electron microscope of corncob and EDTA-GO/ corncob were carried out by an EVO MA10 scanning electron microscope (ZEISS, Germany). Zeta potential of EDTA-corncob and EDTA-GO/corncob were carried out on Zetasizer Nano Series (Malvern, British).

\subsection{Adsorption experiments}

2.4.1 Comparison of adsorption capacity. Seven copies of $0.0100 \mathrm{~g}$ EDTA-GO/corncob were added to $25.00 \mathrm{~mL}$ of methylene blue, crystal violet, acridine orange, methyl red, acid chrome blue $\mathrm{K}$, rhodamine $\mathrm{B}$ and orange IV with initial concentrations $20 \mathrm{mg} \mathrm{L}^{-1}$, respectively. Then, $0.0100 \mathrm{~g}$ of corncob and $0.0100 \mathrm{~g}$ of EDTA-corncob were studied in the same fashion with the above steps. All flasks were shaken at 298 $\mathrm{K}$ to reach adsorption equilibrium. After that, absorbance of methylene blue, crystal violet, acridine orange, methyl red, acid chrome blue $\mathrm{K}$, rhodamine $\mathrm{B}$ and orange IV in supernatant were determined by UV-Vis spectrophotometer at $662 \mathrm{~nm}, 582 \mathrm{~nm}$, $490 \mathrm{~nm}, 435 \mathrm{~nm}, 524 \mathrm{~nm}, 554 \mathrm{~nm}$ and $443 \mathrm{~nm}$, respectively. The adsorption capacity ( $q_{\mathrm{e}}$, expressed in $\mathrm{mg} \mathrm{g}^{-1}$ ) was calculated according to formula (1).

$$
q_{\mathrm{e}}=\frac{\left(C_{0}-C_{\mathrm{e}}\right) \times V}{m}
$$

where $C_{\mathrm{e}}$ is the equilibrium concentration $\left(\mathrm{mg} \mathrm{L}^{-1}\right), C_{0}$ is the initial concentration $\left(\mathrm{mg} \mathrm{L}^{-1}\right), V$ is the solution volume $(\mathrm{L}), m$ is the weight of the adsorbent $(\mathrm{g})$.

2.4.2 Effect of $\mathbf{p H}$ on adsorption capacity. Effect of $\mathrm{pH}$ on adsorption capacity of $0.0100 \mathrm{~g}$ of EDTA/corncob and $0.0100 \mathrm{~g}$ of EDTA-GO/corncob were carried out and the initial concentration of crystal violet was set as $20 \mathrm{mg} \mathrm{L}^{-1}$ with varying $\mathrm{pH}$ at 298 $\mathrm{K}$. After reach to adsorption equilibrium, the other steps were studied in the same fashion with the 2.4.1 comparison of adsorption capacity.

2.4.3 Adsorption kinetics. $0.1000 \mathrm{~g}$ of EDTA-GO/corncob was added to $250 \mathrm{~mL}$ crystal violet solution with initial concentrations $40 \mathrm{mg} \mathrm{L}^{-1}$ at $\mathrm{pH}$ 6.09. The flask was shaken at $298 \mathrm{~K}$ to reach adsorption equilibrium. Then the absorbance of crystal violet in supernatant at different time intervals were determined by UV-Vis spectrophotometer at $582 \mathrm{~nm}$. Each experiment was repeated three times. The capacity of crystal violet adsorbed $\left(q_{t}\right.$, expressed in $\left.\mathrm{mg} \mathrm{g}^{-1}\right)$ was calculated according to the formula (2):

$$
q_{t}=\frac{\left(C_{0}-C_{t}\right) \times V}{m}
$$

where $C_{0}$ and $C_{t}$ are the initial and temporary concentration of the dye $\left(\mathrm{mg} \mathrm{L}^{-1}\right), m$ is the weight of the adsorbent $(\mathrm{g})$, and $V$ is the solution volume (L).

2.4.4 Adsorption isotherm. Adsorption isotherm studies were carried out with $25 \mathrm{~mL}$ of crystal violet with the initial concentrations ranging from 20 to $140 \mathrm{mg} \mathrm{L}^{-1}$. Adsorptions onto $0.0100 \mathrm{~g}$ of EDTA/corncob and $0.0100 \mathrm{~g}$ of EDTA-GO/ corncob were carried out at $298 \mathrm{~K}$ with varying initial concentration at $\mathrm{pH}$ 6.09. After reach to adsorption equilibrium, the other steps are the same as the adsorption kinetics.

The influence of ionic strength on the adsorption of crystal violet on EDTA/corncob and EDTA-GO/corncob was studied in the same fashion with the adsorption isotherm, except that sodium chloride was varied and the initial concentration of crystal violet was set as $40 \mathrm{mg} \mathrm{L}^{-1}$.

2.4.5 Recycling experiments. The adsorption experiment was conducted with initial concentration of crystal violet was set 
as $40 \mathrm{mg} \mathrm{g}^{-1}$ with $0.01 \mathrm{~g}$ of EDTA-GO/corncob at $\mathrm{pH}$ 6.09. After $8 \mathrm{~h}$, the absorbance of crystal violet in supernatant was determined at $582 \mathrm{~nm}$ while the EDTA-GO/corncob was desorbed with ethanol for $5 \mathrm{~h}$. Then EDTA-GO/corncob was filtered and washed repeatedly with distilled water. Finally, they were dried in vacuum oven at $55{ }^{\circ} \mathrm{C}$. Recycling experiments of EDTA/ corncob were studied in the same fashion with EDTA-GO/ corncob. According to the above steps, the adsorption/ desorption were repeated nine times. The capacity of crystal violet adsorbed $\left(q_{\mathrm{e}}\right.$, expressed in $\left.\mathrm{mg} \mathrm{g}^{-1}\right)$ was calculated according to the formula (1).

\section{Results and discussion}

\subsection{Characterization of materials}

Scanning electron microscope (SEM) for corncob and EDTA-GO/ corncob is shown in Fig. 1. The structure of corncob (Fig. 1a and c) and EDTA-GO/corncob (Fig. 1b and d) were clearly revealed by SEM investigations. The SEM micrograph of corncob shows porous surface structure (Fig. 1a and c). Comparing to the SEM images of corncob, EDTA-GO/corncob is mainly lamellar structure.
The FTIR spectra of GO, corncob, EDTA-corncob and EDTA-GO/ corncob are shown in Fig. 2. The characteristic peaks for GO appear at $1110,1580,1650$ and $3450 \mathrm{~cm}^{-1}$ which correspond to stretching vibrations of $\mathrm{C}-\mathrm{O}-\mathrm{C}, \mathrm{C}=\mathrm{C}, \mathrm{C}=\mathrm{O}$ and $-\mathrm{OH}$ bonds, respectively (Fig. 2a). The EDTA-GO/corncob and EDTA-corncob presented peaks for $\mathrm{N}-\mathrm{H}\left(\nu_{\mathrm{N}-\mathrm{H}} 3450 \mathrm{~cm}^{-1}\right), \mathrm{C}=\mathrm{O}\left(\nu_{\mathrm{C}=\mathrm{O}}, 1660 \mathrm{~cm}^{-1}\right), \mathrm{N}-\mathrm{H}\left(\delta_{\mathrm{N}-}\right.$ $\left.\mathrm{H}, 1590 \mathrm{~cm}^{-1}\right)$ and $\mathrm{C}-\mathrm{O}-\mathrm{C}\left(\nu_{\mathrm{C}-\mathrm{O}-\mathrm{C}}, 1110 \mathrm{~cm}^{-1}\right)$, all these functional groups are expected from EDTA and GO (Fig. 2c and d). These peaks at 1110 and $1660 \mathrm{~cm}^{-1}$ are not present in the spectra of unmodified corncob (Fig. 2b), indicating that EDTA and GO are attached successfully on the surface of EDTA-GO/corncob.

\subsection{Adsorption experiment}

3.2.1 Effect of dyes on adsorption capacity. Effect of methylene blue, crystal violet, acridine orange, methyl red, acid chrome blue $\mathrm{K}$, rhodamine $\mathrm{B}$ and orange IV on adsorption capacity of corncob, EDTA-corncob and EDTA-GO/corncob are shown in Fig. 3. It can be seen that corncob, EDTA-corncob and EDTA-GO/corncob can adsorb the seven dyes. Adsorption capacity of the seven dyes on corncob and EDTA-corncob are litter, while adsorption capacity of the seven dyes on EDTA-GO/
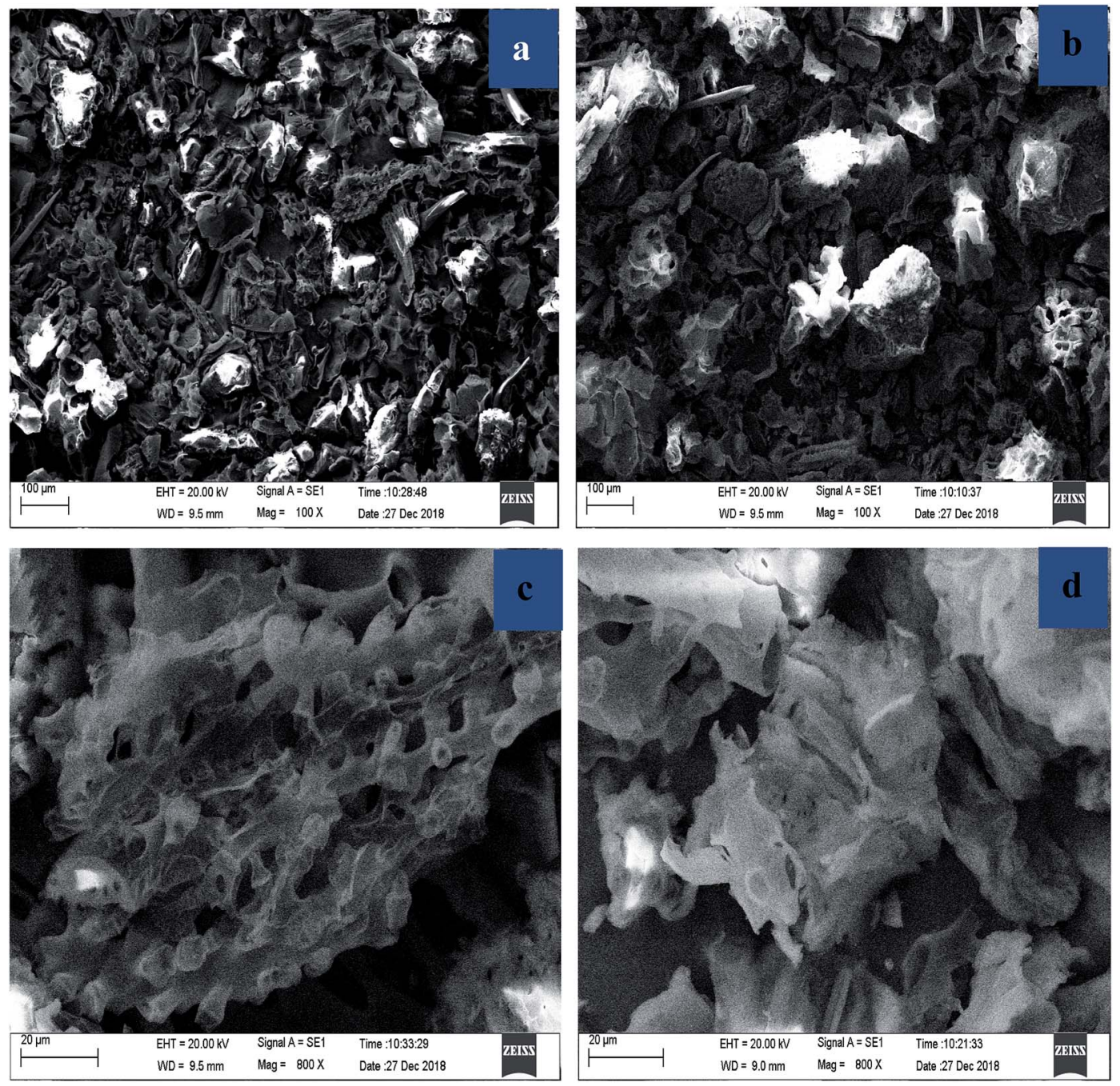

Fig. 1 SEM of corncob ( $a$ and c) and EDTA-GO/corncob (b and d). 


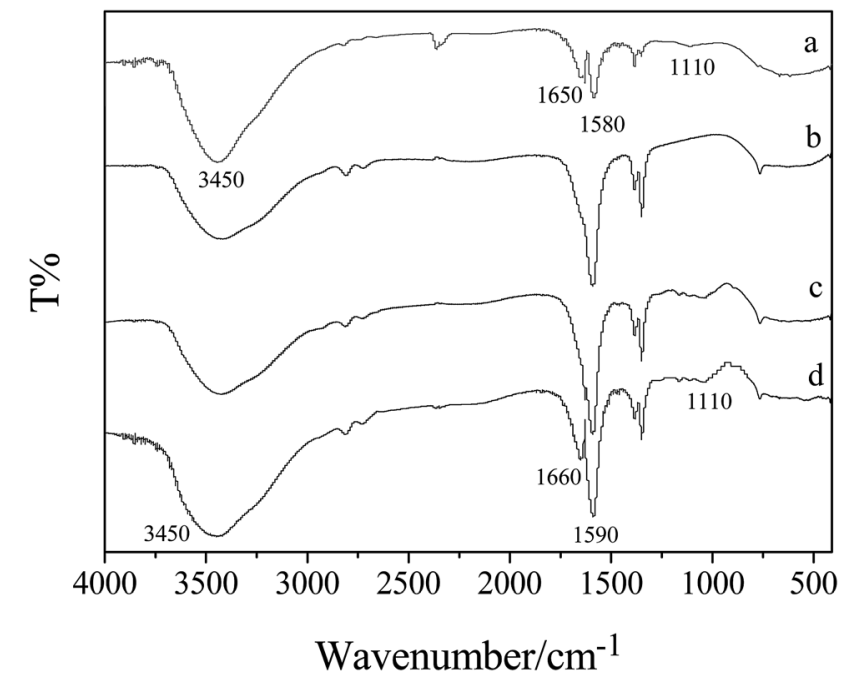

Fig. 2 FTIR spectra of adsorbents. (a) GO; (b) corncob; (c) EDTA/ corncob; (d) EDTA-GO/corncob.

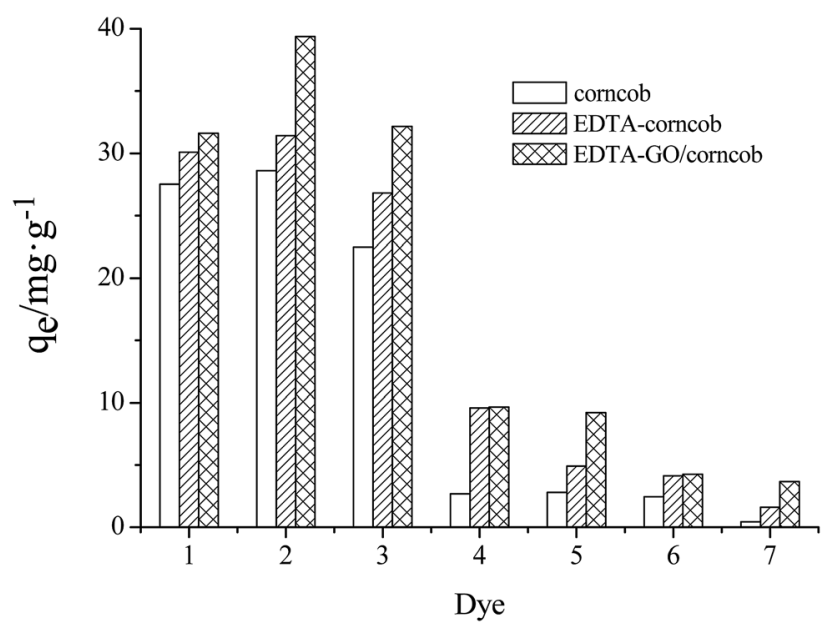

Fig. 3 Comparisons of adsorption capacity $(\mathrm{pH}=7.0)(1)$ methylene blue; (2) crystal violet; (3) acridine orange; (4) methyl red; (5) acid chrome blue K; (6) rhodamine B; (7) orange IV.

corncob are higher than corncob and EDTA-corncob. Especially, the adsorption capacity of crystal violet on EDTA-GO/corncob is the most. So crystal violet was chosen as the object dye in the following experiments. Chemical structure of crystal violet is shown in Fig. 4.<smiles>CN(C)c1ccc(C(=C2C=CC(=[N+](C)C)C=C2)c2ccc(N(C)C)cc2)cc1</smiles>

Fig. 4 Chemical structure of crystal violet.
3.2.2 Adsorption kinetics. Fig. 5 showed the adsorption capacity of crystal violet on EDTA-corncob and EDTA-GO/ corncob versus adsorption time at $298 \mathrm{~K}$. According to the kinetics data (Fig. 5), the adsorption capacity of crystal violet on EDTA-corncob and EDTA-GO/corncob increased very fast at 0$100 \mathrm{~min}$ and reached equilibrium at $200 \mathrm{~min}$.

In order to explain the adsorption mechanism, the adsorption kinetic data of crystal violet on EDTA-corncob and EDTA$\mathrm{GO} /$ corncob were treated with pseudo-first-order and pseudosecond-order models, as shown in Fig. S1 and S2 (ESI $\dagger$ ), and the experimental data was listed in Table 1.

The pseudo first-order kinetics model (eqn (3)) and the pseudo-second-order kinetics model (eqn (4)) can be expressed as follows:

$$
\begin{gathered}
\log \left(q_{\mathrm{e}}-q_{t}\right)=\log q_{\mathrm{e}}-K_{1} \frac{t}{2.303} \\
\frac{t}{q_{t}}=\frac{1}{K_{2} q_{\mathrm{e}}{ }^{2}}+\frac{t}{q_{\mathrm{e}}}
\end{gathered}
$$

where $q_{\mathrm{e}}$ and $q_{t}$ are the capacity of the dye adsorbed on the adsorbents at equilibrium and at time $t\left(\mathrm{mg} \mathrm{g}^{-1}\right)$, respectively, $K_{1}\left(\mathrm{~min}^{-1}\right)$ and $K_{2}\left(\mathrm{~g} \mathrm{mg}{ }^{-1} \mathrm{~min}^{-1}\right)$ are the rate constant of pseudo-first-order and pseudo-second-order kinetics models.

As listed in Table 1, the adsorption kinetics of EDTA-corncob and EDTA-GO/corncob were nicely described by pseudo-secondorder model $(R=0.99)$ and the calculated $q_{\mathrm{e}}$ was very close to the experimental $q_{\mathrm{e}}$. This indicated that chemisorption or chemical bonding between active sites of EDTA-corncob and EDTA-GO/ corncob and crystal violet might dominate the adsorption process.

3.2.3 Adsorption isotherms. The adsorption isotherms of crystal violet on EDTA-corncob and EDTA-GO/corncob were studied at $298 \mathrm{~K}$ in Fig. 6 . The adsorption capacity of the crystal violet on EDTA-corncob and EDTA-GO/corncob reaches the maximum as the initial concentration of the crystal violet increase gradually as shown in Fig. 6.

The experimental adsorption equilibrium results on EDTAcorncob and EDTA-GO/corncob were fitted by the Langmuir model (eqn (5) and (6)), Freundlich isotherm model (eqn (7))

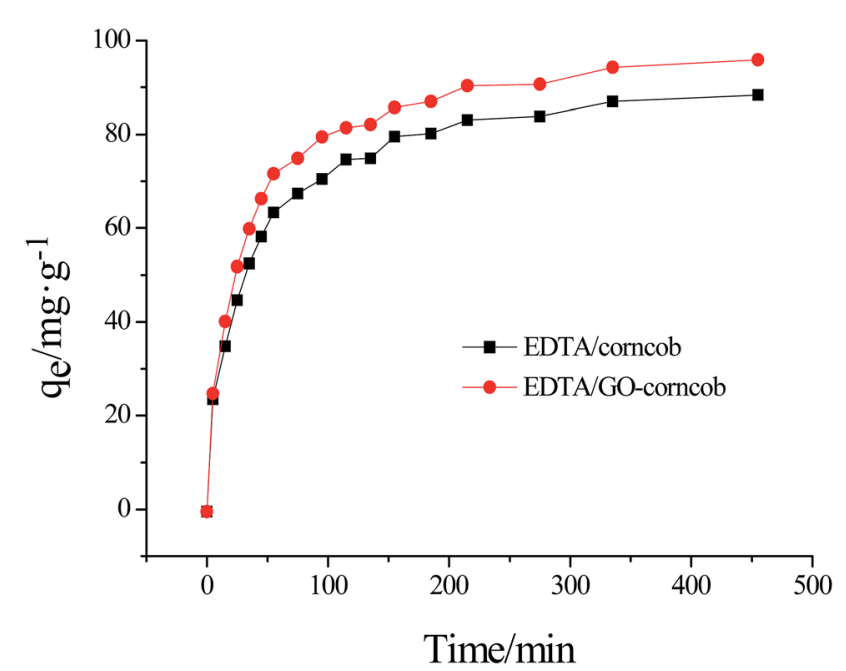

Fig. 5 Adsorption kinetics. 
Table 1 Kinetic parameters for the adsorption of crystal violet on EDTA/corncob and EDTA-GO/corncob at $298 \mathrm{~K}$

\begin{tabular}{|c|c|c|c|c|c|c|c|}
\hline Adsorbent & $q_{\text {eqex }} / \mathrm{mg} \mathrm{g}^{-1}$ & \multicolumn{3}{|c|}{ Pseudo-first-order kinetics model } & \multicolumn{3}{|c|}{ Pseudo-second-order kinetics model } \\
\hline EDTA-GO/cornob & 95.87 & 0.1566 & 71.12 & 0.9421 & 0.05171 & 95.24 & 0.9981 \\
\hline
\end{tabular}

and Sips model (eqn (8)). The Langmuir model base on three assumptions: $:^{28}$ (1) the adsorption of molecule is a monolayer adsorption; (2) the adsorption of adsorbent surface is uniform; (3) there is no interaction among adsorbed molecules. The Freundlich model assumes that the adsorption surface is heterogeneous, that interactions between adsorbed molecules can occur, and that multilayer adsorption is possible. Sips model is an improved Langmuir model.

$$
\begin{gathered}
q_{\mathrm{e}}=\frac{q_{\mathrm{m}} C_{\mathrm{e}}}{K_{\mathrm{d}}+C_{\mathrm{e}}} \\
\frac{C_{\mathrm{e}}}{q_{\mathrm{e}}}=\frac{1}{q_{\mathrm{m}} K_{\mathrm{L}}}+\frac{C_{\mathrm{e}}}{q_{\mathrm{m}}} \\
\log q_{\mathrm{e}}=\log K_{\mathrm{F}}+\left[\frac{1}{n}\right] \log C_{\mathrm{e}} \\
q_{\mathrm{e}}=\frac{q_{\mathrm{m}} K_{\mathrm{S}} C_{\mathrm{e}}^{m_{\mathrm{S}}}}{1+K_{\mathrm{S}} C_{\mathrm{e}}{ }^{m_{\mathrm{S}}}}
\end{gathered}
$$

where $q_{\mathrm{e}}, C_{\mathrm{e}}, K_{\mathrm{L}}, q_{\mathrm{m}}$ and $K_{\mathrm{d}}$ are the equilibrium adsorption capacity $\left(\mathrm{mg} \mathrm{g}^{-1}\right)$, the equilibrium concentration $\left(\mathrm{mg} \mathrm{L}^{-1}\right)$, Langmuir adsorption constant, the maximum adsorption capacity $\left(\mathrm{mg} \mathrm{g}^{-1}\right)$ and effective dissociation constant. $K_{\mathrm{F}}$ is the Freundlich adsorption coefficient, which is related to the dosage and properties of adsorbent, the properties and temperature of adsorbate, $n$ is the Freundlich constant, which is related to the nature of adsorption system. $K_{\mathrm{S}}$ is the Sips adsorption coefficient, $m_{\mathrm{S}}$ is the Sips constant, which indicates the heterogeneity of adsorbent. The closer the $m_{\mathrm{S}}$ value is to 1 , the more homogeneous the adsorbent surface is. ${ }^{29}$

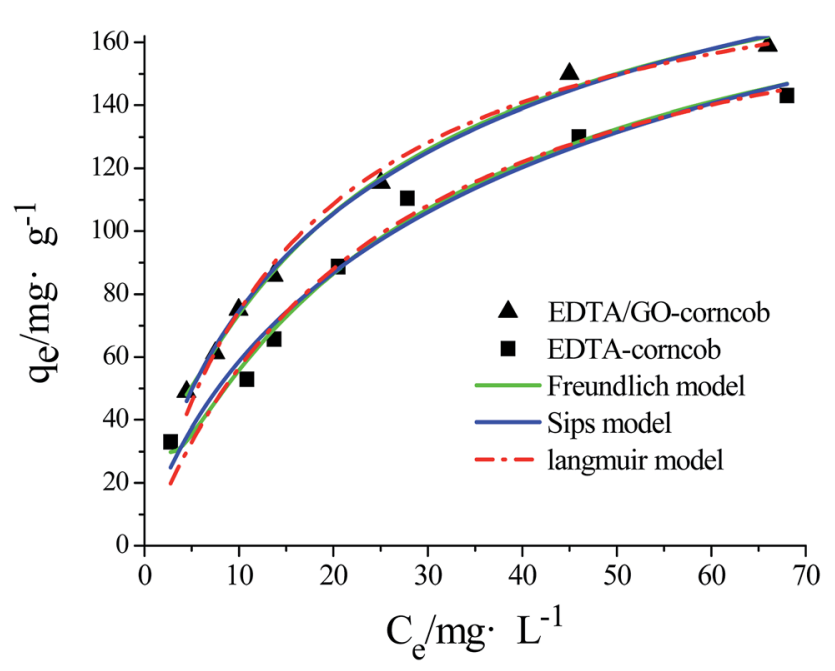

Fig. 6 Adsorption isotherms.
The Freundlich model provided a slightly better fit for the adsorption data of crystal violet onto EDTA-corncob and EDTAGO/corncob (Table 2) than Langmuir model and Sips model. Therefore, the adsorption of crystal violet onto EDTA-corncob and EDTA-GO/corncob is heterogeneous adsorptions.

A comparison of the maximum adsorption capacities $\left(q_{\mathrm{m}}\right)$ of EDTA-corncob and EDTA-GO/corncob for crystal violet are listed in Table 3 with literature values of $q_{\mathrm{m}}$ of other adsorbents. It can be seen that the maximum adsorption capacities of EDTAcorncob and EDTA-GO/corncob for crystal violet are higher than that of other materials which might be attributed to the higher specific surface area of graphene and the EDTA groups.

\subsection{Adsorption mechanism}

The $\mathrm{pH}$ of solution affects the surface charge of the adsorbents as well as the degree of ionization of pollutants. Change of $\mathrm{pH}$ value affects the adsorptive process through dissociation of functional groups on the adsorbent surface active site. ${ }^{38}$

The impact of initial $\mathrm{pH}$ in the range of 2.0-10.0 on crystal violet adsorption by the EDTA/corncob and EDTA-GO/corncob are shown in Fig. 7a. The highest adsorption capacities of crystal violet on EDTA/corncob and EDTA-GO/corncob were detected at $\mathrm{pH}$ of 6.0. The adsorption of crystal violet on EDTA/ corncob and EDTA-GO/corncob are pH dependent, the adsorption capacity of crystal violet on EDTA/corncob and EDTA-GO/ corncob increases with the increase of $\mathrm{pH}(\mathrm{pH}<6.0)$, but the adsorption capacity of crystal violet on EDTA/corncob and EDTA$\mathrm{GO} /$ corncob decreases with the increase of $\mathrm{pH}(\mathrm{pH}>6.0)$.

The results may be attributed to the electrostatic attraction between the anions on the adsorbent and the cations on the dye. Fig. $7 \mathrm{~b}$ showed the zeta potentials of EDTA/corncob and EDTA-GO/corncob. As shown in Fig. 7b, the isoelectric point $\left(\mathrm{pH}_{\mathrm{zpc}}\right)$ of EDTA/corncob and EDTA-GO/corncob are 2.05 and 1.98 , respectively. At low $\mathrm{pH}(\mathrm{pH}>2.05)$, the surface of EDTA/ corncob is negative charge because of the $-\mathrm{COO}^{-}$groups on the surface of EDTA/corncob. At low pH ( $\mathrm{pH}>1.98)$, the surface of EDTA-GO/corncob is negative charge, which is attributed to more ${ }^{-\mathrm{COO}^{-}}$groups. The negative charge on the surface of EDTA/corncob and EDTA-GO/corncob increases from $\mathrm{pH} 2.0$ to 6.0 and then decreases with the increase of $\mathrm{pH}$, and reaches the maximum at $\mathrm{pH}$ 6.0. The surface of acridine orange is positive charge. At $\mathrm{pH}$ around 6.0, the electrostatic interaction between crystal violet and EDTA/corncob or EDTA-GO/corncob is strongest, so the adsorption capacity of crystal violet on the absorbents reaches the maximum.

The effect of ionic strength on the adsorption of crystal violet on EDTA/corncob or EDTA-GO/corncob were studied by carried out a series of experiments at $\mathrm{NaCl}$ solutions $(0.00,0.02,0.04$, 
Table 2 Adsorption isotherm parameters of crystal violet onto on EDTA/corncob and EDTA-GO/corncob at $298 \mathrm{~K}$

\begin{tabular}{|c|c|c|c|c|c|c|c|c|c|}
\hline Adsorbent & \multicolumn{3}{|c|}{ Langmuir model } & \multicolumn{3}{|c|}{ Freundlich model } & \multicolumn{3}{|c|}{ Sips model } \\
\hline EDTA-GO/cornob & 203.9 & 16.54 & 0.9929 & 22.91 & 1.985 & 0.9982 & 0.03865 & 0.5864 & 0.9981 \\
\hline
\end{tabular}

Table 3 Comparison of maximum adsorption capacities of different adsorbents (298 K)

\begin{tabular}{|c|c|c|c|}
\hline Adsorbent & Dye & $q_{\mathrm{m}} / \mathrm{mg} \mathrm{g}^{-1}$ & Ref. \\
\hline AC-AgNPLs & Crystal violet & 87.2 & 30 \\
\hline Chitosan/nanodiopside nanocomposite & Crystal violet & 104.66 & 31 \\
\hline ZVI-GAM & Crystal violet & 172.41 & 33 \\
\hline Chitin nanowhiskers & Crystal violet & 39.56 & 34 \\
\hline Zinc oxide nanorods loaded on activated carbon & Crystal violet & 113.64 & 35 \\
\hline EDTA/corncob & Crystal violet & 185.2 & This work \\
\hline EDTA-GO/cornob & Crystal violet & 203.9 & This work \\
\hline
\end{tabular}
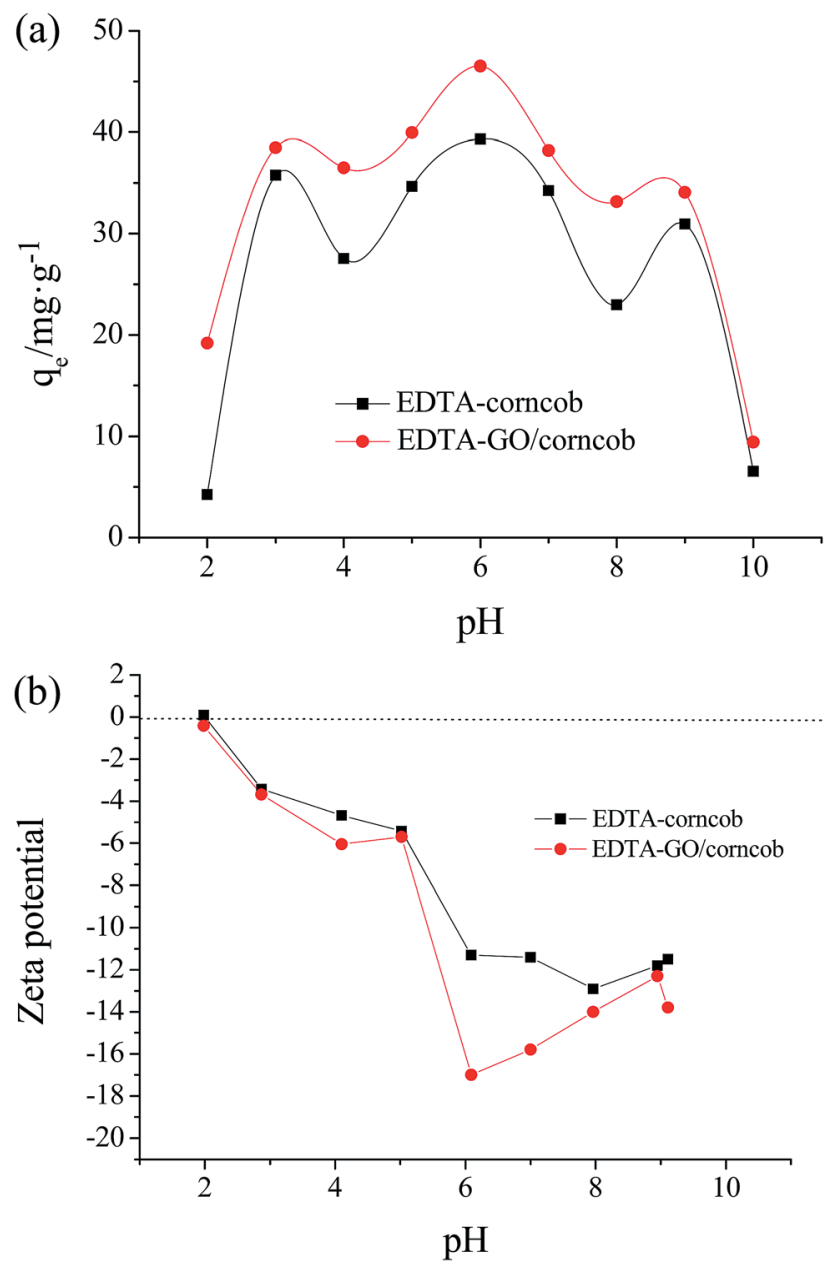

Fig. 7 (a) Effect of $\mathrm{pH}$ on the adsorption capacity. (b) Zeta potential at the different $\mathrm{pH}$.
0.06, $0.08,0.10 \mathrm{~mol} \mathrm{~L}^{-1}$ ), and solution $\mathrm{pH}$ was adjusted to 6.0 before adsorption. The results are shown in Fig. 8. It is observed that the adsorption capacity of crystal violet on EDTA/corncob or EDTA-GO/corncob under the condition of $0.10 \mathrm{M} \mathrm{NaCl}$ remains $81.31 \%$ and $82.63 \%$ that of without addition of $\mathrm{NaCl}$. The adsorption processes of crystal violet on EDTA/corncob or EDTA-GO/corncob are dependent on $\mathrm{NaCl}$ concentrations, which showed that electrostatic attraction dominates the adsorption process. The interaction mechanisms of crystal violet adsorption with EDTA/corncob and EDTA-GO/corncob were showed in Fig. 9.

\subsection{Adsorbent recycling}

Taking into account the practical application, the adsorption capacity and the reusability property are two key parameters to

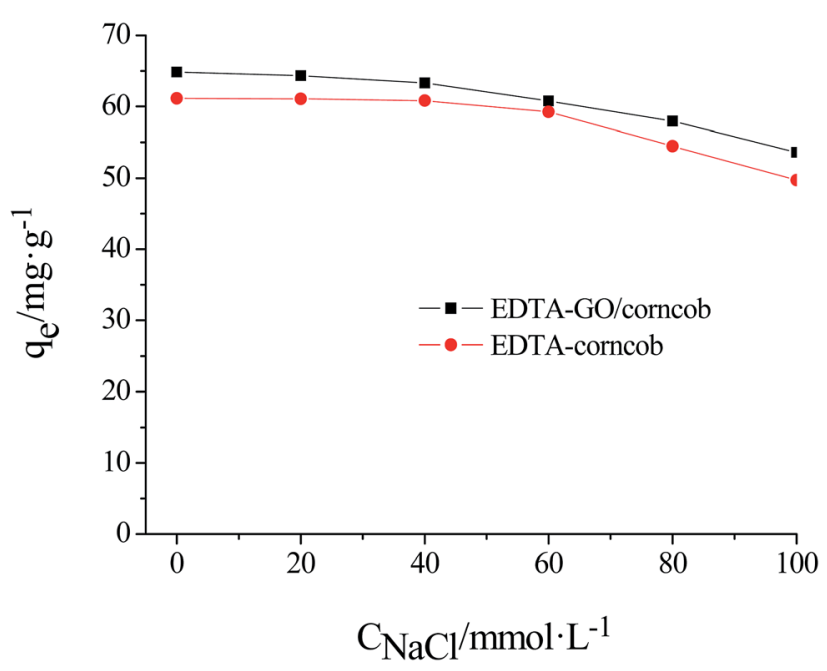

Fig. 8 Effect of $\mathrm{NaCl}$ on the adsorption capacity. 


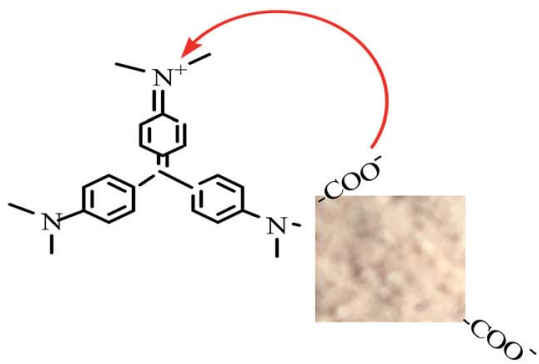

EDTA/corncob

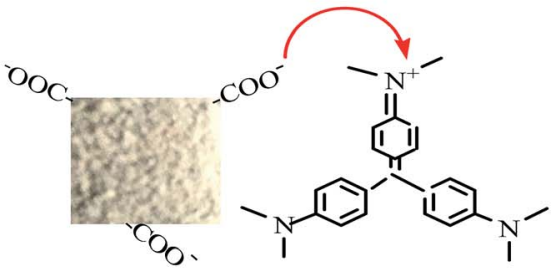

EDTA-GO/corncob

Fig. 9 The interaction mechanisms.

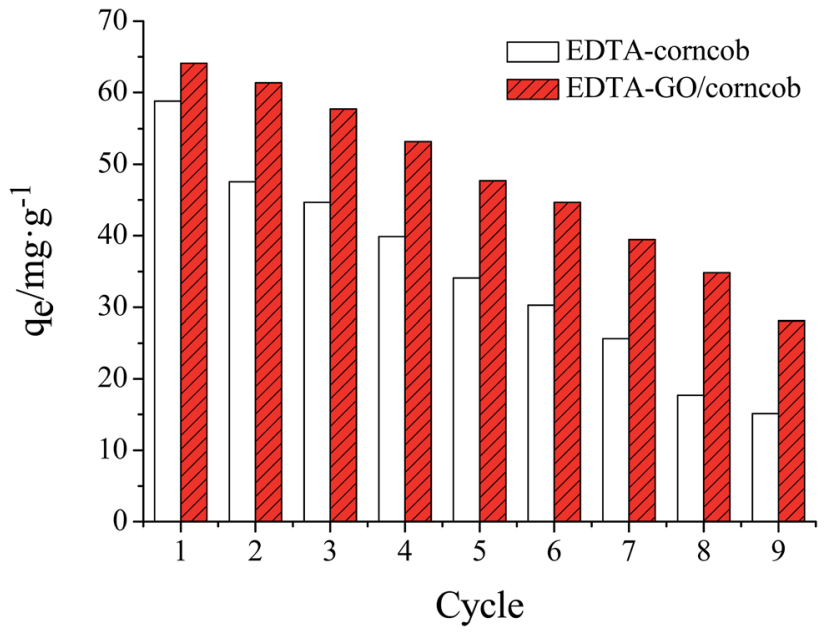

Fig. 10 Reusability of EDTA/corncob and EDTA-GO/corncob.

evaluate an adsorbent. An ideal adsorbent should not only possess higher adsorption capability, but also show better reusability property, which will significantly reduce the overall cost for the adsorbents. ${ }^{39}$

Reusability of EDTA/corncob or EDTA-GO/corncob is shown in Fig. 10. The crystal violet solutions with the concentration of $40 \mathrm{mg} \mathrm{\textrm {L } ^ { - 1 }}$ were prepared and EDTA/corncob or EDTA-GO/ corncob was recycled for nine times to investigate the adsorption cycle number of EDTA/corncob or EDTA-GO/corncob. At the first adsorption equilibrium, the maximum adsorption capacity of EDTA/corncob or EDTA-GO/corncob was 58.8 and $64.1 \mathrm{mg} \mathrm{g}^{-1}$, respectively. With the increase of cycle number, the adsorption capacity showed a trend of decreasing. The adsorption capacity of EDTA/corncob was $30.3 \mathrm{mg} \mathrm{g}^{-1}$ at the sixth adsorption, while the maximum adsorption capacity of EDTA-GO/corncob is $28.1 \mathrm{mg} \mathrm{g}^{-1}$ at the ninth adsorption. This result showed that EDTA/corncob can be reused for five times and EDTA-GO/corncob can be reused for eight times.

\section{Conclusions}

In summary, EDTA/corncob and EDTA-GO/corncob were prepared successful and could be used as an efficient and recyclable adsorbent for crystal violet. The adsorption capacity of crystal violet on EDTA-GO/corncob is higher than EDTA/ corncob. The most suitable $\mathrm{pH}$ of adsorption of crystal violet on EDTA-GO/corncob was around 6.0. The adsorption equilibration time was 200 minutes. The adsorption kinetics fit well with pseudo-second-order model and the adsorption isotherm fit well with Freundlich isotherm model. Adsorption mechanism of crystal violet on EDTA/corncob and EDTA-GO/corncob were electrostatic attraction. EDTA/corncob can be reused for five times and EDTA-GO/corncob can be reused for eight times. The facile regeneration of EDTA-GO/corncob definitely could reduce the operating cost. We believe that EDTA-GO/corncob will have potentially wide application in the removal of dye pollutants from aquatic systems.

\section{Conflicts of interest}

There are no conflicts to declare.

\section{Acknowledgements}

This work was supported by the Nation Natural Science Foundation of China (No. 21703189), Xianyang Science and Technology Research Project of China (No. 2018k02-20), Shaanxi Province Innovation and Entrepreneurship Project for College Students of China in 2017 (No. 2502) and Xianyang Normal University Innovation and Entrepreneurship Project for College Students of China in 2017 (No. 2017070).

\section{References}

1 M. G. Dosskey, Environ. Manage., 2001, 28, 577-598.

2 A. J. Jafari, B. Kakavandi, R. R. Kalantary, H. Gharibi, A. Asadi, A. Azari, A. A. Babaei and A. Takdastan, Korean J. Chem. Eng., 2016, 33(10), 2878-2890.

3 K. Shakir, A. F. Elkafrawy, H. F. G. Shokry, G. E. Beheir and M. Refaat, Water Res., 2010, 44(5), 1449-1461.

4 H. Zhang, Q. Luan, H. Tang, F. Huang, M. Zheng, Q. Deng, X. Xiang, C. Yang, J. Shi, C. Zheng and Q. Zhou, Cellulose, 2017, 24(2), 903-914.

5 J. Núñez, M. Yeber, N. Cisternas, R. Thibaut, P. Medina and C. Carrasco, J. Hazard. Mater., 2019, 371, 705-711. 
6 K. Sirirerkratana, P. Kemacheevakul and S. Chuangchote, J. Cleaner Prod., 2019, 215, 123-130.

7 Q. Y. Yue, B. Y. Gao, Y. Wang, H. Zhang, X. Sun, S. G. Wang and R. R. Gu, J. Hazard. Mater., 2008, 152(1), 221-227.

8 J. Liang, X. A. Ning, J. Sun, J. Song, Y. Hong and H. Cai, J. Cleaner Prod., 2018, 204, 12-19.

9 I. Chaari, E. Fakhfakh, M. Medhioub and F. Jamoussi, J. Mol. Struct., 2019, 1179, 672-677.

10 N. Nikooe and E. Saljoughi, Appl. Surf. Sci., 2017, 413, 41-49. 11 J. Zhao, Z. Zou, R. Ren, X. Sui, Z. Mao, H. Xu, Y. Zhong, L. Zhang and B. Wang, Eur. Polym. J., 2018, 108, 212-218.

12 N. A. H. M. Zaidi, L. B. L. Lim and A. Usman, Environmental Technology \& Innovation, 2019, 13, 211-223.

13 L. Dai, W. Zhu, L. He, F. Tan, N. Zhu, Q. Zhou, M. He and G. Hu, Bioresour. Technol., 2018, 267, 510-516.

14 L. Zhou, H. Zhou, Y. Hu, S. Yan and J. Yang, J. Environ. Manage., 2019, 234, 245-252.

15 Y. Song, J. Tan, G. Wang and L. Zhou, Carbohydr. Polym., 2018, 199, 178-185.

16 J. Wu, T. Zhang, C. Chen, L. Feng, X. Su, L. Zhou, Y. Chen, A. Xia and X. Wang, Bioresour. Technol., 2018, 266, 134-138.

17 N. Abidi, J. Duplay, A. Jada, E. Errais, M. Ghazi, K. Semhi and M. Trabelsi-Ayadi, C. R. Chim., 2019, 22(2-3), 113-125.

18 S. Shakoor and A. Nasar, Groundwater for Sustainable Development, 2017, 5, 152-159.

19 H. Ma, J. B. Li, W. W. Liu, M. Miao, B. J. Cheng and S. W. Zhu, Bioresour. Technol., 2015, 190, 13-20.

20 M. T. Vu, H. P. Chao, T. V. Trinh, T. T. Le, C. C. Lin and H. N. Tran, J. Cleaner Prod., 2018, 180, 560-570.

21 H. Lin, S. Han, Y. Dong and Y. He, Appl. Surf. Sci., 2017, 412, 152-159.

22 D. Lv, Y. Liu, J. Zhou, K. Yang, Z. Lou, S. A. Baig and X. Xu, Appl. Surf. Sci., 2018, 428, 648-658.

23 D. Wu, L. Hu, Y. Wang, Q. Wei, L. Yan, T. Yan, Y. Li and B. Du, J. Colloid Interface Sci., 2018, 523, 56-64.
24 E. F. C. Chaúque, L. N. Dlamini, A. A. Adelodun, C. J. Greyling and J. C. Ngila, Physics and Chemistry of the Earth, Parts $A / B / C, 2017,100,201-211$.

25 Y. Yang, S. Song and Z. Zhao, Colloids Surf., A, 2017, 513, 315-324.

26 M. Lv, L. Yan, C. Liu, C. Su, Q. Zhou, X. Zhang, Y. Lan, Y. Zheng, L. Lai, X. Liu and Z. Ye, Chem. Eng. J., 2018, 349, 791-799.

27 L. Sun, H. Yu and B. Fugetsu, J. Hazard. Mater., 2012, 203204, 101-110.

28 X. Xin, W. Si, Z. Yao, R. Feng, B. Du, L. Yan and Q. Wei, J. Colloid Interface Sci., 2011, 359, 499-504.

29 G. McKay, A. Mesdaghinia, S. Nasseri, M. Hadi and M. S. Aminabad, Chem. Eng. J., 2014, 251, 236-247.

30 A. H. AbdEl-Salam, H. A. Ewais and A. S. Basaleh, J. Mol. Liq., 2017, 248, 833-841.

31 S. G. Nasab, A. Semnani, A. Teimouri, M. J. Yazd, T. M. Isfahani and S. Habibollahi, Int. J. Biol. Macromol., 2019, 124, 429-443.

32 G. Sharma, A. Kumar, M. Naushad, A. García-Peñas, A. H. AlMuhtaseb, A. A. Ghfar, V. Sharma, T. Ahamad and F. J. Stadler, Carbohydr. Polym., 2018, 202, 444-453.

33 J. Liu, Y. Wang, Y. Fang, T. Mwamulima, S. Song and C. Peng, J. Mol. Liq., 2018, 250, 468-476.

34 S. Gopi, A. Pius and S. Thomas, Journal of Water Process Engineering, 2016, 14, 1-8.

35 E. A. Dil, M. Ghaedi, A. Ghaedi, A. Asfaram, M. Jamshidi and M. K. Purkait, J. Taiwan Inst. Chem. Eng., 2016, 59, 210-220.

36 C. Muthukumaran, S. V. Murugaiyan and M. Thirumarimurugan, J. Taiwan Inst. Chem. Eng., 2016, 63, 354-362.

37 G. Wen and Z. G. Guo, Colloids Surf., A, 2018, 541, 58-67.

38 C. Yang, L. Lei, P. Zhou, Z. Zhang and Z. Lei, J. Colloid Interface Sci., 2015, 443, 97-104.

39 H. T. Xing, J. H. Chen, X. Sun, Y. H. Huang, Z. B. Su, S. R. Hu, W. Weng, S. X. Li, H. X. Guo, W. B. Wu, Y. S. He, F. M. Li and Y. Huang, Chem. Eng. J., 2015, 263, 280-289. 